\title{
CO Outflows in Low-Mass Protostar Candidates in Taurus
}

\author{
M. Tamura (JPL, NAOJ), N. Ohashi (NRO), G. Moriarty-Schieven (DRO) \\ M. Hayashi (Univ. Tokyo), N. Hirano (Hitotsubashi Univ.)
}

The. Taurus dark cloud is one of the nearest active sites of low-mass star formation. The IRAS satellite has discovered numerous low-luminosity far-infrared sources in this cloud, some of which are completely invisible at optical wavelengths. There are several arguments suggesting that the invisible sources are low-mass protostars which are younger than $\mathrm{T}$ Tauri stars and powered by the accretion of infalling gas and dust (Adams, Lada, \& Shu 1987; Beichman et ai. 1986; Myers et al. 1987; Kenyon et al. 1990)

Tamura et al. (1991) made a near-infrared imaging survey of a complete, flux-limited sample of cold IRAS sources in the Taurus dark cloud, of which 8 are optical T Tauri-like objects and 16 are optically invisible sources. They identified all 24 sources with nearinfrared counterparts; one is entirely nebulous without a point-like source, and the others generally have an unresolved peak with or without an extended component at $2.2 \mu \mathrm{m}$. $75 \%$ of the 24 sample sources are associated with infrared/optical nebulosity about 1000 $\mathrm{AU}$ to $10000 \mathrm{AU}$ in size. A number of the sources show a clear bipolar or monopolar morphology, suggestive of a close relation of the nebulosity with a bipolar mass outflow; the nebulosity is due to scattering of light from the central source by the dust associated with the mass outflow extending to the poles of a circumstellar dust disk.

The association of such compact molecular outflow has also been confirmed by a CO $\mathrm{J}=3-2$ outflow survey of the same sample at CSO (Moriarty-Schieven et al. 1992).

However, the $\mathrm{CO}$ observations were made only toward the central source, and the near-infrared observations did not provide kinematical information. Therefore, we are currently conducting a $\mathrm{CO} \mathrm{J=1-0}$ outflow mapping survey of the same sample with the Nobeyama Millimeter Array. In this paper, we report a successful detection and mapping of an outflow associated one of the sample sources, IRAS $04365+2535$.

The observations of IRAS $04365+2535$ were made with the D-configuration of the NMA. The resultant resolution was $9^{\prime \prime} .1 \times 5^{\prime \prime} .4$ at a position angle of $147^{\circ}$.

The map of the blue- and red-shifted components are shown in Fig. $1 \mathrm{a}$ and $1 \mathrm{~b}$. Each component is clearly separated and centered around the central near-infrared source: the blue-shifted component is extending predominantly to the north-west $\left(\sim 45^{\prime \prime}\right)$, showing a cometary structure with its apex near the near-infrared source, while the red-shifted component is extending to the south-east $\left(\sim 30^{\prime \prime}\right)$, although the latter is less extensive. This compact outflow is mapped for the first time and was not revealed by an outflow survey using a single dish telescope (Heyer et al. 1987), although the presence of the outflow has been suggested from a snapshot of the OVRO interferometer (Telebey et al. 1989 ) and the Nobeyama $45 \mathrm{~m}$ telescope observations (Hirano et al. in prep.). Apparently, a high resolution is crucial to detect and map such outflows in these cold IRAS sources.

Fig. 2 is a logarithmic contour map of IR.AS $04365+2535$ taken with the infrared imager on the Kitt Peak $1.3 \mathrm{~m}$ telescope. The pixel scale is $1^{\prime \prime} .3$ pixel $^{-1}$ and the field of 
view is $78^{\prime \prime} \times 83^{\prime \prime}$. It is evident that an extensive faint infrared nebulosity is associated around a bright point-like central source. It is noteworthy that the infrared nebula is extending roughly in the same direction as the $\mathrm{CO}$ bipolar outflow, coincident with the blue-shifted component of the outflow. This is consistent with the interpretation that the IR nebula is associated with the bipolar outflow. The absence of the infrared nebula in the direction of the blue-shifted component is probably due to the dust obscuration by a hypothetical disk around the central source extending perpendicular to the $\mathrm{CO}$ outflow. Our CS $\mathrm{J}=2$-1 observations (Ohashi et al. in prep.) did delineate such disk-like structure around the infrared source.

References

Adams, F.C. et al. 1987, ApJ, 312, 788; Beichman, C.A. et al. 1986, ApJ, 307, 337

Heyer, M.H. 1987, ApJ, 321, 370; Kenyon, S.J. et al. 1990, AJ, 99, 869

Moriarty-Schieven, G.H. et al. 1992, ApJ, in press; Myers, P.C. et al. 1987, ApJ, 319, 340

Tamura, M. et al. 1991, ApJ, 374, L25; Telebey, S. et al. 1989, ApJ, 340, 472

Figs. 1: NMA CO outflow map of IRAS $04365+2535$, the blue-shifted component ( $1 \mathrm{a}:$ left) and the red-shifted component ( $1 \mathrm{~b}:$ right). The northern cross represents the $2.2 \mu \mathrm{m}$ source position and the southern one $98 \mathrm{GHz}$ continuum peak position.

Fig. 2: $K$-band $(2.2 \mu \mathrm{m}$ ) image of $I R A S 04365+2535$ (in logarithmic scale).
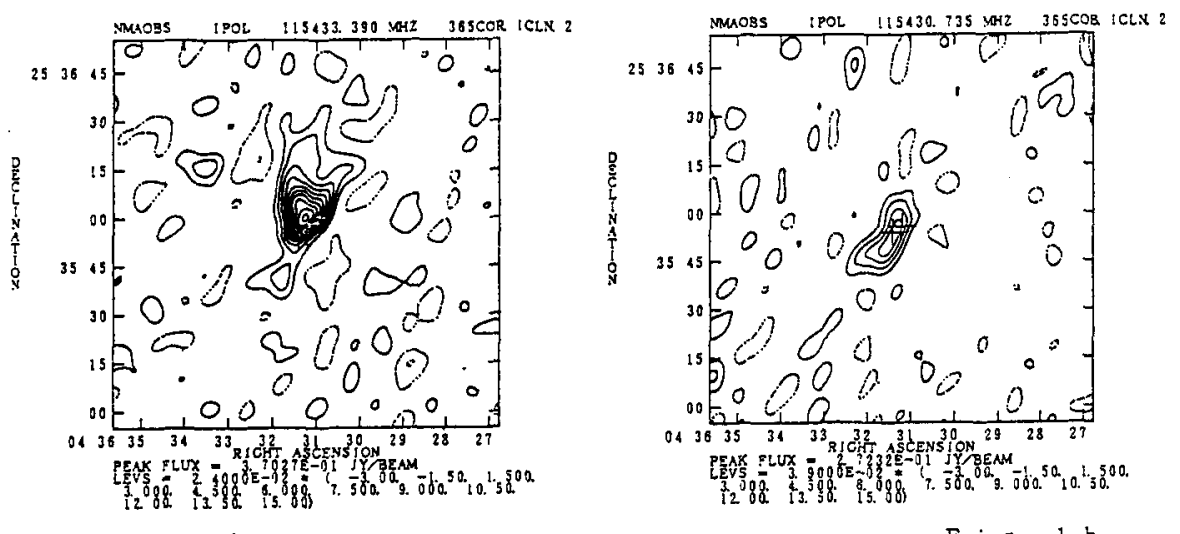

F i g. 1 a

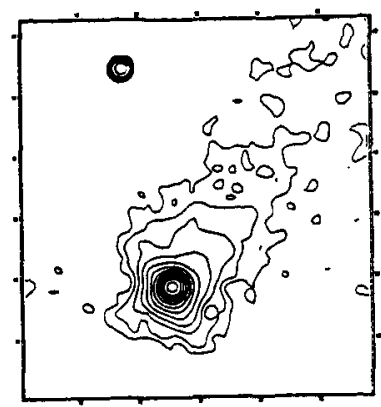

F i g. 2 